\title{
Infective endocarditis by an unusual organism - Abiotrophia species
}

\author{
Sudusinghe $\mathrm{DH}^{1}$, Liyanage $\mathrm{A}^{2}$, Marasinghe $\mathrm{C}^{2}$, Chandrasiri $\mathrm{S}^{3}$ \\ ${ }^{I}$ Department of Physiology, ${ }^{2}$ Department of Medicine, Faculty of Medical Sciences, University of Sri \\ Jayewardenepura, Sri Lanka. ${ }^{3}$ Colombo South Teaching Hospital, Kalubowila, Sri Lanka.
}

Correspondence: Dr. Dinesha Himali Suduinghe

e-mail: dinesha94@yahoo.com

\section{Introduction}

Infective endocarditis (IE) is one of potentially life threatening condition presenting with pyrexia of unknown origin. The commonest organism of IE in Sri Lanka is viridans streptococcus species (1). We report an uncommon bacterium causing IE, which is Abiotrophia species and we were unable to find previous records of IE caused by this organism.

Abiotrophia is also known as nutritionally variant streptococci (NDS). It is a part of oral, upper respiratory tract and urogenital flora (2). Infective endocarditis due to this organism is associated with high rates of treatment failure, relapses and mortality than Viridans streptococci (3). It is a slow growing organism with special nutritional requirements and challenging phenotype testing. Hence it is a major cause for culture negative infective endocarditis $(3,4)$. Therefore an early identification is necessary to reduce morbidity and mortality.

\section{Case report}

A 48-year-old male presented with a history of intermittent fever for three months. One week before admission he had an episode of right ankle joint pain and swelling, which settled with analgesics. He also had loss of appetite, fatigue and weight loss.There was no past history of rheumatic fever. He denied recent history of dental extractions, intravenous cannulation, substance abuse or catheterization.

On admission he was febrile, pale and had first degree finger clubbing. A pan systolic murmur was heard at the mitral area suggestive of a mitral regurgitation without evidence of pulmonary hypertension or heart failure. Rest of the clinical examination was unremarkable. Full blood count showed neutrophil leukocytosis and normochromic normocytic anaemia. Urine analysis showed field full of red cells. Ultrasound scan of abdomen revealed $5 \mathrm{~mm}$ calculus in the right kidney. ESR was $100 \mathrm{~mm}$ and CRP was $45.9 \mathrm{mg} / \mathrm{L}$. Transthoracic echocardiogram revealed a mass attached to the posterior mitral valve (Figure 1) with normal left ventricular function.

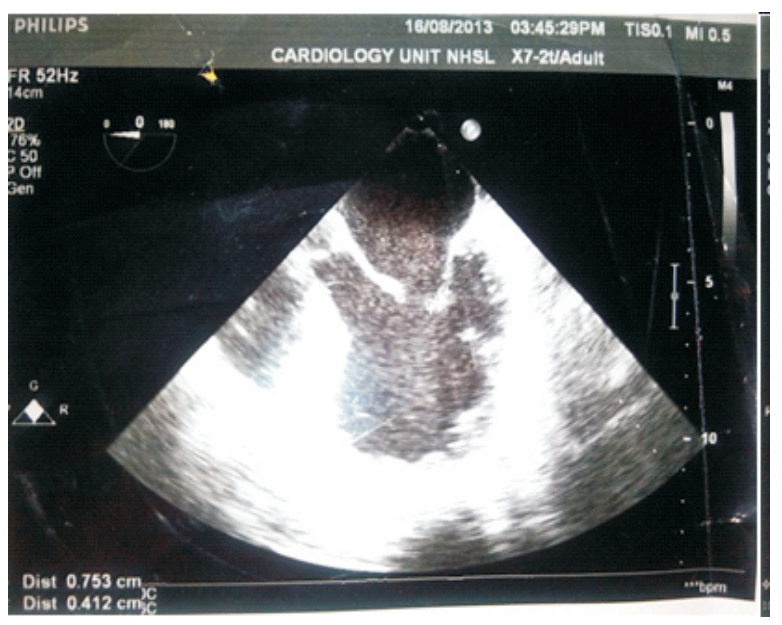

Figure 1: Trans esophageal Echocardiogram of the patient showing the healed vegetation

Three blood cultures were positive for the same organism after 24 hour manual sub culture. A minute colony appeared on blood agar without evidence of haemolysis. But it did not grow on MacConkey agar. The colony did not show carbon dioxide dependency. It showed gram positive cocci smaller than usual streptococci with short chains and Lancefield grouping did not give agglutination. Since this organism was fastidious, antibiotic sensitivity was done on both chocolate and blood agar. Both ABSTs did not show sensitivity zone for ceftriaxone but penicillin gave a good zone diameter making penicillin the choice of treatment. 
In Clinical and Laboratory Standard Institute (CLSI) test method there is no interpretation criterion for this type of organism given in M100. It was sub cultured in CLED medium and the organism grew in larger colony size. Because of this, the isolated organism was assumed to be cysteine dependent. There were no centers with biochemical test kits (ex: API step) that can identify streptococci species in Sri Lanka.

Our patient was initially started on ceftriaxone, before the transthoracic echocardiogram after taking blood cultures. As we suspected infection with Abiotrophia species, he was started on penicillin and gentamicin. Antibiotics were continued for six weeks. Fever responded well and inflammatory markers were normalized. Patient went home after full course of antibiotics without complications.

\section{Discussion}

Abiotrophia is a rare cause for IE which accounts for $5-6 \%$ of all causes of IE in developed countries (3). Abiotrophia is a cause of serious infections including endocarditis, brain abscess and septic arthritis and known to associate with higher morbidity and mortality $(3,5)$. One of the serious complications of IE due to this infection is destructive valvular lesions causing heart failure in which early surgical intervention should be considered. Slow growth of the organism and need of unique nutritional requirement for growth make the isolation of the organism difficult. However, early antibiotic treatment with penicillin and gentamicin gives a high rate of success. Although infective endocarditis caused by Abiotrophia is extremely rare, early identification is crucial to improve the outcome of the patients as treatment with ceftriaxone leads to treatment failure.

\section{Conclusion}

It is possible to isolate Abiotrophia species even with manual blood culture system. As this organism can cause complications and treatment failure, attempts should be taken to take blood cultures before starting antibiotics and it is important to know when to suspect this organism in the blood culture since rapid identification of this organism is not available in Sri Lanka.

\section{References}

1. Piyasiri DLB, Chandrasiri P. Prospective study of patients with infective endocarditis. Bulletin of the Sri Lanka College of Microbiologist, 2011; 9: 22-3.

2. Christensen JJ, Facklam RR. Granulicatella and Abiotrophia species from human clinical specimen. J Clin Microbiol, 2001; 39: 3520-3.

3. Brouqui P, Raoult D. Endocarditis due to rare and fastidious bacteria. Clin Microbiol Rev, 2001; 14(1): 177-207.

4. Frenkle A, Hirsh W. Spontaneous development of L forms of streptococci requiring secretions of other bacteria or sulfhydryl compounds for normal growth. Nature, 1961; 191: 728-30.

5. Cerceo E, Christie JD, Nachamkin I, Lautenbach E. Central nervous system infections due to Abiotrophia and Granulicatella species: an emerging challenge? Diagn Microbiol Infect Dis, 2004; 48(3): 161-5. 